\title{
ON COMPLETENESS OF RANDOM TRANSITION COUNT FOR MARKOV CHAINS
}

\author{
A. PALMA \\ Received December 7, 2004 and, in revised form, November 24, 2005
}

\begin{abstract}
It is shown that the random transition count is complete for Markov chains with a fixed length and a fixed initial state, for some subsets of the set of all transition probabilities.
\end{abstract}

\section{Introduction}

Consider the set $\mathbf{X}$ of all trajectories of Markov chains with a finite state space $S=\{1, \ldots, n\}$, with a fixed length $N \geq 2$ and a fixed initial state $x_{1}=i^{\prime}$. Let $\mathbf{x}=\left(x_{1}, \ldots, x_{N}\right) \in \mathbf{X}$ denote such trajectory. The probability distributions on $\mathbf{X}$ can be parametrized by stochastic matrices. We denote them by

$$
\mathcal{P}=\left\{p=\left(p_{i, j}\right): \underset{i, j \in S}{\forall} p_{i, j} \geq 0, \quad \sum_{j=1}^{n} p_{i, j}=1 \text { for each } i \in S\right\} .
$$

2000 Mathematics Subject Classification. Primary 62B05.

Key words and phrases. Markov chain, random transition count, sufficient statistic, minimal sufficient statistic, complete statistic.

ISSN 1425-6908（C) Heldermann Verlag. 
Let $\mathcal{Z} \subset S \times S$ denote a fixed subset satisfying

$$
\underset{i \in S j \in S}{\exists}(i, j) \notin \mathcal{Z}
$$

We denote by $\mathcal{P}_{\mathcal{Z}}$ the space of stochastic matrices $p \in \mathcal{P}$, such that

$$
\underset{(i, j) \in \mathcal{Z}}{\forall} p_{i, j}=0 \text {. }
$$

Explicitly, a probability distribution on $\mathbf{X}$ is given by the formula

$$
P_{\left(p_{i, j}\right)}(\{\mathbf{x}\})=p_{x_{1}, x_{2}} \cdot \ldots \cdot p_{x_{N-1}, x_{N}} \quad \text { for } \mathbf{x}=\left(x_{1}, \ldots, x_{N}\right) \in \mathbf{X} .
$$

Throughout the paper we are dealing with a statistical space of the form $\left(\mathbf{X},\left\{P_{\left(p_{i, j}\right)}:\left(p_{i, j}\right) \in \mathcal{P}_{\mathcal{Z}}\right\}\right)$. Obviously, the assumption $p \in \mathcal{P}_{\mathcal{Z}}$ means that the transitions in one step from $i$ to $j$ are forbidden for $(i, j) \in \mathcal{Z}$. The space $\mathcal{P}_{\mathcal{Z}}$ remains nonempty because of (1).

It is worth noting that a number of important types of Markov chains can be characterized by suitable classes $\mathcal{P}_{\mathcal{Z}}$. Some classes of random walks and Markov chains of order $r>1$ described in [6], can be mentioned as examples.

The random transition count $F$ is defined in the usual way as a matrix

$$
F(\mathbf{x})=\left(f_{i, j}\right)_{i, j=1, \ldots, n}, \quad f_{i, j}=\#\left\{t=1, \ldots, N-1: x_{t}=i, x_{t+1}=j\right\}
$$

for $i, j \in S$. Obviously $F$ is a basic tool in any statistical investigation. In particular $F$ is a sufficient statistic. It turns out that $F$ is also complete if all matrices from $\mathcal{P}$ are allowed, as was shown by Denny and Yakowitz [1], Denny and Wright [2]. Moreover the statistic $F$ is always complete for "Markov bridge", when the initial and the final state are fixed (see [6]).

Complete statistics are one of the fundamental concepts in mathematical statistics. In particular these play the essential role in the theory of uniformly most powerful unbiased tests and in minimum variance unbiased estimation. An exposition of this theory can be found in [3] and [4], [5].

Unfortunately, the random transition count $F$ could be non-complete for some space $\mathcal{P}_{\mathcal{Z}}$. The suitable examples are given at the end of this section. Thus it is necessary to make some extra assumptions about the set $\mathcal{Z}$ (cf. condition (9) in Section 3). As the main result, given in Section 3 , we describe some classes of sets $\mathcal{Z}$, such that $F$ is a complete statistic for distributions parametrized by matrices from $\mathcal{P}_{\mathcal{Z}} \subset \mathcal{P}$. In Section 2 we introduce some natural classification of the states of the Markov chain for the class $\mathcal{P}_{\mathcal{Z}}$ and we give some auxiliary results (Lemmas 2.1-2.4). The proofs of the lemmas are based on some combinatorical tools.

The following example is remarkable and can be found in [6], with a different proof.

Proposition 1.1. Let $S=\{1,2,3,4,5\}, N=5$ and let $x_{1}=1$ be fixed. For some space $\mathcal{P}_{\mathcal{Z}}$ the statistic $F$ is not complete. 
Proof. Let the set $\mathcal{Z}$ be defined by

$$
(S \times S) \backslash \mathcal{Z}=\{(1,2),(1,3),(2,4),(2,5),(3,4),(3,5),(4,1),(5,1)\} .
$$

Then any matrix $p \in \mathcal{P}_{\mathcal{Z}}$ can be written as

$$
p=\left[\begin{array}{ccccc}
0 & q & 1-q & 0 & 0 \\
0 & 0 & 0 & r & 1-r \\
0 & 0 & 0 & s & 1-s \\
1 & 0 & 0 & 0 & 0 \\
1 & 0 & 0 & 0 & 0
\end{array}\right], \quad q, r, s \in[0,1] .
$$

The statistic $F(\mathbf{x})=\left(f_{i, j}\right)$ takes on the following values with corresponding probabilities

$$
\begin{array}{lc}
M_{1}=\left[\begin{array}{lllll}
0 & 1 & 1 & 0 & 0 \\
0 & 0 & 0 & 1 & 0 \\
0 & 0 & 0 & 0 & 0 \\
1 & 0 & 0 & 0 & 0 \\
0 & 0 & 0 & 0 & 0
\end{array}\right], & M_{2}=\left[\begin{array}{lllll}
0 & 1 & 1 & 0 & 0 \\
0 & 0 & 0 & 0 & 1 \\
0 & 0 & 0 & 0 & 0 \\
0 & 0 & 0 & 0 & 0 \\
1 & 0 & 0 & 0 & 0
\end{array}\right], \\
P\left(F(\mathbf{x})=M_{1}\right)=q \cdot(1-q) \cdot r, & P\left(F(\mathbf{x})=M_{2}\right)=q \cdot(1-q) \cdot(1-r), \\
M_{3}=\left[\begin{array}{lllll}
0 & 1 & 1 & 0 & 0 \\
0 & 0 & 0 & 0 & 0 \\
0 & 0 & 0 & 1 & 0 \\
1 & 0 & 0 & 0 & 0 \\
0 & 0 & 0 & 0 & 0
\end{array}\right], & M_{4}=\left[\begin{array}{lllll}
0 & 1 & 1 & 0 & 0 \\
0 & 0 & 0 & 0 & 0 \\
0 & 0 & 0 & 0 & 1 \\
0 & 0 & 0 & 0 & 0 \\
1 & 0 & 0 & 0 & 0
\end{array}\right] \\
P\left(F(\mathbf{x})=M_{3}=q \cdot(1-q) \cdot s,\right. & P\left(F(\mathbf{x})=M_{4}\right)=q \cdot(1-q) \cdot(1-s), \\
P\left(F(\mathbf{x})=M_{7}\right)=(1-q) &
\end{array}
$$


Then the expectation $\mathbb{E}(g \circ F)$ vanishes for any non-zero function $g$ satisfying

$$
\begin{aligned}
& g\left(M_{1}\right)=g\left(M_{2}\right)=-g\left(M_{3}\right)=-g\left(M_{4}\right), \\
& g\left(M_{5}\right)=g\left(M_{6}\right)=-\left(\frac{1-q}{q}\right)^{2} \cdot g\left(M_{7}\right)=-\left(\frac{1-q}{q}\right)^{2} \cdot g\left(M_{8}\right) .
\end{aligned}
$$

Moreover, note that if the initial state $x_{1}$ is not fixed, then the statistic $F$ is not sufficient. The natural sufficient statistic which should be investigated in such a situation is

$$
G(\mathbf{x})=\left(x_{1}, F(\mathbf{x})\right),
$$

but in general $G$ is not complete.

Proposition 1.2. Let $S=\{1,2\}, N=2$. The statistic $G\left(x_{1}, x_{2}\right)=$ $\left(x_{1},\left(f_{i, j}\right)\right)$ is sufficient, but not complete.

Proof. Indeed, for a fixed initial distribution $\left(p_{1}, p_{2}\right) ; p_{1}, p_{2} \in[0,1], p_{1}+$ $p_{2}=1$ and for any transition probability matrix

$$
p=\left[\begin{array}{ll}
p_{1,1} & p_{1,2} \\
p_{2,1} & p_{2,2}
\end{array}\right] \in \mathcal{P},
$$

the statistic $G\left(x_{1}, x_{2}\right)=\left(x_{1},\left(f_{i, j}\right)\right)$ takes on the following values with corresponding probabilities

$$
\begin{array}{ll}
G_{1}=\left(1,\left[\begin{array}{ll}
1 & 0 \\
0 & 0
\end{array}\right]\right), & P\left(F=G_{1}\right)=p_{1} \cdot p_{1,1}, \\
G_{2}=\left(1,\left[\begin{array}{ll}
0 & 1 \\
0 & 0
\end{array}\right]\right), & P\left(F=G_{2}\right)=p_{1} \cdot p_{1,2}, \\
G_{3}=\left(2,\left[\begin{array}{ll}
0 & 0 \\
1 & 0
\end{array}\right]\right), & P\left(F=G_{3}\right)=p_{2} \cdot p_{2,1}, \\
G_{4}=\left(2,\left[\begin{array}{ll}
0 & 0 \\
0 & 1
\end{array}\right]\right), & P\left(F=G_{4}\right)=p_{2} \cdot p_{2,2} .
\end{array}
$$

Then the expectation $\mathbb{E}_{p}(g \circ G)$ vanishes if only the function $g$ satisfies $g\left(G_{1}\right)=g\left(G_{2}\right)=p_{2}, g\left(G_{3}\right)=g\left(G_{4}\right)=-p_{1}$. 


\section{The classification of states and auxiliary results}

Let us fix a set $\mathcal{Z}$ satisfying (1). For $p \in \mathcal{P}_{\mathcal{Z}}$ (cf. (2)), we use standard notations $\left(p_{i, j}(n)\right)_{i, j \in S}=p(n)=p^{n}$. To simplify our considerations, let us observe that there exists a matrix $p^{\mathcal{Z}} \in \mathcal{P}_{\mathcal{Z}}$ with a maximal set of positive elements:

$$
p_{i, j}^{\mathcal{Z}}>0 \text { if only }(i, j) \notin \mathcal{Z} \text {. }
$$

We say that a state $i$ is inessential for the class $\mathcal{P}_{\mathcal{Z}}$ if

$$
\underset{j}{\exists}\left[\left(\underset{t_{0} \geq 1}{\exists} \quad p_{i, j}^{\mathcal{Z}}\left(t_{0}\right)>0\right) \wedge\left(\underset{t \geq 1}{\forall} p_{j, i}^{\mathcal{Z}}(t)=0\right)\right] .
$$

A state is essential if it is not inessential. We define an equivalence relation in the class of essential states: $i \sim j$ if

$$
\underset{s, t \geq 1}{\exists}\left(p_{i, j}^{\mathcal{Z}}(s)>0 \wedge p_{j, i}^{\mathcal{Z}}(t)>0\right) .
$$

Consequently the set of essential states for the class $\mathcal{P}_{\mathcal{Z}}$ splits into classes of equivalence. We denote them by $S_{1}, S_{2}, \ldots, S_{\beta_{0}}, \beta_{0} \in \mathbb{N}$.

For the sake of completeness we prove the following auxiliary results, similar to lemma of Denny, Wright (cf. [2]). For any matrix $f$ of dimension $n \times n$, let $f^{\prime}=\left(f_{i, j}^{\prime}\right)$ denote a "table" being the matrix $f$ with deleted elements $f_{i, i+1}, \quad i=1, \ldots, n\left(f_{n, n+1} \equiv f_{n, 1}\right)$. More precisely

$$
f_{i, j}^{\prime}=f_{i, j} \quad \text { for } j \neq i+1(\bmod n)
$$

and

$$
f^{\prime}=\left(f_{i, j}^{\prime}\right)_{i=1, \ldots, n ; j=1, \ldots, i, i+2, \ldots, n}
$$

Lemma 2.1. Fix $i^{\prime}, i^{\prime \prime} \in S$ and $N \geq 2$. Let $\mathcal{M}_{N-1}^{i^{\prime}, i^{\prime \prime}}$ denote a set of matrices $f$ of dimension $n \times n$ satisfying

(i) $\sum_{i, j} f_{i, j}=N-1$,

(ii) $\quad \sum_{j} f_{i, j}+\delta_{i^{\prime \prime}}(i)=\sum_{j} f_{j, i}+\delta_{i^{\prime}}(i) \quad$ for $i \in S$.

The function $f \mapsto f^{\prime}$ defined by $(3),\left(3^{\prime}\right)$ is one-one on the class $\mathcal{M}_{N-1}^{i^{\prime}, i^{\prime \prime}}$. There exist functions $\phi_{i}^{i^{\prime}, i^{\prime \prime}}$ on tables $f^{\prime}$ satisfying $\left(3^{\prime}\right)$, such that

$$
\phi_{i}^{i^{\prime}, i^{\prime \prime}}\left(f^{\prime}\right)=f_{i, i+1} \quad \text { for } i \in S .
$$

Proof. By elementary (but tedious) calculations, the equations (i), (ii) give in particular

$$
f_{1,2}=\frac{1}{n}\left(N-1-\sum_{i, j \neq i+1} f_{i, j}^{\prime}-(n-1) \cdot r_{2}\left(f^{\prime}\right)-\ldots-r_{n}\left(f^{\prime}\right)+\left(i^{\prime}-i^{\prime \prime}\right)\right)
$$


with

$$
r_{i}\left(f^{\prime}\right)=\left(\sum_{j \neq i-1} f_{j, i}^{\prime}-\sum_{j \neq i+1} f_{i, j}^{\prime}\right) \quad \text { for } i=2, \ldots, n .
$$

Next, we define by induction the functions $\left(\phi_{i}^{i^{\prime}, i^{\prime \prime}}\right)$ on $f^{\prime}$, as follows

$$
\left\{\begin{array}{l}
\phi_{1}^{i^{\prime}, i^{\prime \prime}}\left(f^{\prime}\right)=f_{1,2} \\
\phi_{i}^{i^{\prime}, i^{\prime \prime}}\left(f^{\prime}\right)=\phi_{i-1}^{i^{\prime}, i^{\prime \prime}}\left(f^{\prime}\right)+r_{i}\left(f^{\prime}\right)+\delta_{i^{\prime}}(i)-\delta_{i^{\prime \prime}}(i)
\end{array}\right.
$$

Lemma 2.2. Fix $i^{\prime} \in S$ and $N \geq 2$. Let $\mathcal{M}_{N-1}^{i^{\prime}}$ denote a set of matrices $f$ of dimension $n \times n$ with integer elements satisfying

(I) $\quad \sum_{i, j} f_{i, j}=N-1$,

(II) there exists $i^{\prime \prime} \in S$ such that

$$
\sum_{j} f_{i, j}+\delta_{i^{\prime \prime}}(i)=\sum_{j} f_{j, i}+\delta_{i^{\prime}}(i) \quad \text { for } i \in S .
$$

The function $f \mapsto f^{\prime}$ defined by (3), $\left(3^{\prime}\right)$ is one-one on the class $\mathcal{M}_{N-1}^{i^{\prime}}$. There exist functions $\phi_{i}^{i^{\prime}}$ with integer values, such that

$$
\phi_{i}^{i^{\prime}}\left(f^{\prime}\right)=f_{i, i+1} \quad \text { for } i \in S,
$$

for $f^{\prime}$ satisfying $\left(3^{\prime}\right)$ and (3).

Proof. Let $f$ be a matrix with integer elements satisfying conditions (i) and (ii) from Lemma 2.1, with some $i^{\prime \prime} \in S$. Let us define numbers (cf. (5))

$$
f_{1,2}^{i^{\prime \prime \prime}}=\frac{1}{n}\left(N-1-\sum_{i, j \neq i+1} f_{i, j}^{\prime}-(n-1) \cdot r_{2}\left(f^{\prime}\right)-\ldots-r_{n}\left(f^{\prime}\right)+\left(i^{\prime}-i^{\prime \prime \prime}\right)\right)
$$

with $r_{i}\left(f^{\prime}\right)$ given by the formula (6). By the same elementary calculations as in the proof of the previous lemma, we obtain, putting $i^{\prime \prime \prime}=i^{\prime \prime}, \quad f_{1,2}^{i^{\prime \prime}}=f_{1,2}$. Moreover, for any $i^{\prime \prime \prime} \neq i^{\prime \prime}$, we have $f_{1,2}^{i^{\prime \prime \prime}} \notin \mathbb{Z}$.

Now assume that a matrix $f$ wifh integer elements satisfies (I) and (II). Define once more $f_{1,2}^{i^{\prime \prime \prime}}$ by formula $\left(5^{\prime}\right)$ for $i^{\prime \prime \prime}=1, \ldots, n$. Then there exists exactly one number $i_{0}^{\prime \prime \prime}$ such that $f_{1,2}^{i_{0}^{\prime \prime \prime}} \in \mathbb{Z}$. Thus we have $f_{1,2}=f_{1,2}^{i_{0}^{\prime \prime \prime}}$ (and moreover (ii) is satisfied for $\left.i^{\prime \prime}=i_{0}^{\prime \prime \prime}\right)$. 
Now $\phi_{1}^{i^{\prime}}\left(f^{\prime}\right)$ is defined as $f_{1,2}^{i_{0}^{\prime \prime \prime}}$. Other values $\phi_{i}^{i^{\prime}}\left(f^{\prime}\right)$ are obtained by induction:

$$
\left\{\begin{array}{l}
\phi_{1}^{i^{\prime}}\left(f^{\prime}\right)=f_{1,2}^{i_{0}^{\prime \prime \prime}} \\
\phi_{i}^{i^{\prime}}\left(f^{\prime}\right)=\phi_{i-1}^{i^{\prime}}\left(f^{\prime}\right)+r_{i}\left(f^{\prime}\right)+\delta_{i^{\prime}}(i)-\delta_{i_{0}^{\prime \prime \prime}}(i)
\end{array}\right.
$$

in the way analogical to (7).

Corollary 2.3. The function $f \mapsto f^{\prime}$ defined by $(3),\left(3^{\prime}\right)$ is one-one on the class $F[\mathbf{x}]$ of the random transition counts $F$ for the trajectories $\mathbf{x} \in \mathbf{X}$ with a fixed initial state $x_{1}=i^{\prime}$ and a fixed length $N \geq 2$.

The following lemma was used (in almost the same form) by Denny and Wright [2]. Assume that integers $n \geq 1, m(1) \geq 1, \ldots, m(n) \geq 1$ and $q \geq 0$ are fixed. Denote by $A$ the set of systems of non-negative integers $z=\left(z_{i, j}\right)$, $1 \leq i \leq n, 1 \leq j \leq m(i)$ satisfying

$$
\sum_{i=1}^{n} \sum_{j=1}^{m(i)} z_{i, j} \leq q
$$

Fix real $c>0$. Denote by $B$ the set of all systems of positive numbers $y=\left(y_{i, j}\right), \quad 1 \leq i \leq n, 1 \leq j \leq m(i)$ such that

$$
\sum_{j=1}^{m(i)} y_{i, j} \leq c \quad \text { for any } 1 \leq i \leq n .
$$

Let $\varphi_{i}: A \rightarrow\{0,1,2, \ldots\}, i=1, \ldots, n$. For each $z \in A$ let us define functions $W_{z}(y)$, as follows

$$
W_{z}(y)=\prod_{i=1}^{n}\left[\prod_{j=1}^{m(i)} y_{i, j}^{z_{i, j}} \cdot\left(c-y_{i, 1}-\ldots-y_{i, m(i)}\right)^{\varphi_{i}\left(\left(z_{i, j}\right)\right)}\right] .
$$

Lemma 2.4. The system of functions $W_{z}: B \rightarrow \mathbb{R}$, indexed by $z \in A$, is linearly independent.

Proof. Cf. Lemma 2 in [2] or Lemma 3.7 in [6]. 


\section{The main result}

Let $\mathcal{Z} \in S \times S$ be a fixed set. We always assume that $\mathcal{Z}$ satisfies (1), that is

$$
\underset{i \in S}{\forall} \underset{j \in S}{\exists}(i, j) \notin \mathcal{Z}
$$

Recall that using $\mathcal{Z}$ one can naturally define the class $S_{0}$ of inessential states and classes of equivalence $S_{1}, \ldots, S_{\beta_{0}}, \beta_{0} \in \mathbb{N}$, in essential states.

To prove the completeness of the statistic $F$, we need some more special properties of the set $\mathcal{Z}$. Let us remark that, by Proposition 1.1, such additional assumptions about the set $\mathcal{Z}$ are necessary.

In the paper we construct the set $\mathcal{Z}$ such that the state space $S$ will be the whole class of essential states. Thus in what follows, we assume that there exists a permutation $\left(\pi_{1}, \pi_{2}, \ldots, \pi_{n}\right)$ of the set $S$, satisfying

$$
\left\{\left(\pi_{1}, \pi_{2}\right), \ldots,\left(\pi_{n-1}, \pi_{n}\right),\left(\pi_{n}, \pi_{1}\right)\right\} \cap \mathcal{Z}=\emptyset .
$$

Theorem 3.1. Let $\mathcal{Z}$ satisfy (1) and (9). Let $\left(\mathbf{X},\left\{P_{\left(p_{i, j}\right)}:\left(p_{i, j}\right) \in \mathcal{P}_{\mathcal{Z}}\right\}\right)$, with $\mathcal{P}_{\mathcal{Z}}$ given by (2), be the statistical space of all trajectories of Markov chains with the state space $S=\{1, \ldots, n\}$, a fixed initial state $x_{1}=i^{\prime}$, and the trajectory size $N \geq 2$. Then the random transition count $F$ is complete.

As the sufficency of $F$ is obvious, from Bahadur's Theorem [7] we have

Corollary 3.2. Under the assumptions of Theorem 3.1, $F$ is the minimal sufficient statistic.

Proof of Theorem 3.1. Let $\left(\pi_{1}, \pi_{2}, \ldots, \pi_{n}\right)$ denote any permutation satisfying condition (9). It is obvious, that by changing suitably the notation, one can assume that $\left(\pi_{1}, \pi_{2}, \ldots, \pi_{n}\right)=(1, \ldots, n)$. Thus we can assume that

$$
\{(1,2), \ldots,(n-1, n),(n, 1)\} \cap \mathcal{Z}=\emptyset .
$$

Let us remind that for a trajectory $\mathbf{x}$ of size $N \geq 2$, the initial state $x_{1}$ is fixed. A random transition count of the trajectory is denoted by $f=\left(f_{i, j}\right)$ for $i, j \in S$. Moreover, let $f^{\prime}$ denote the matrix $f$ with deleted elements $f_{i, i+1}, i=1, \ldots, n,($ cf. (3), (3')).

For any $\left(p_{i, j}\right) \in \mathcal{P}_{\mathcal{Z}}$, the distribution of $F$ is given by

$$
P_{\left(p_{i, j}\right)}(F=f)=\xi\left(x_{1}, f\right) \cdot \prod_{i=1}^{n} \prod_{j=1}^{n} p_{i, j}^{f_{i, j}},
$$

where $\xi\left(x_{1}, f\right)=\#\{\mathbf{x}: F(\mathbf{x})=f\}$ denotes the number of corresponding trajectories. 
To show that the statistic $F$ is complete, it is enough to prove that condition

$$
\sum_{f} d(f) \cdot \xi\left(x_{1}, f\right) \cdot \prod_{i=1}^{n} \prod_{j=1}^{n} p_{i, j}^{f_{i, j}}=0 \quad \text { for each }\left(p_{i, j}\right) \in \mathcal{P}_{\mathcal{Z}}
$$

implies that

$$
d(f)=0 \quad \text { for each } f .
$$

For any table $f^{\prime}$, (cf. Section 2), let $W_{f^{\prime}}(p)$ denote the function defined by (8) on the set $\left\{\left(p_{i, j}\right), 1 \leq i, j \leq n,(i, j) \notin \mathcal{Z}: \sum_{j} p_{i, j} \leq 1\right.$ for $\left.1 \leq i \leq n\right\}$. After change of indices $i, j$, we can rewrite this set, as follows

$$
\left\{\left(p_{i, j}\right): 1 \leq i \leq n, 1 \leq j \leq m(i), j \neq i+1(\bmod n)\right\} .
$$

Then

$$
W_{f^{\prime}}(p)=\prod_{i=1}^{n}\left(\prod_{\substack{j=1 \\ j \neq i+1}}^{m(i)} p_{i, j}^{f_{i, j}^{\prime}} \cdot\left(1-\sum_{\substack{j=1 \\ j \neq i+1}}^{m(i)} p_{i, j}\right)^{\phi_{i}\left(f^{\prime}\right)}\right),
$$

where $\phi_{i}, i=1, \ldots, n$ are functions ocurring in Lemma 2.1, Lemma 2.2 and Corollary 2.3. Using the notation $d\left(f^{\prime}\right)=\xi\left(x_{1}, f\right) \cdot d(f)$, the equality (11) can be written as

$$
\sum_{f^{\prime}} d\left(f^{\prime}\right) \cdot W_{f^{\prime}}(p)=0
$$

for any $p=\left(p_{i, j}\right)_{1 \leq i \leq n, 1 \leq j \leq m(i), j \neq i+1(\bmod n)}$ from the open set

$$
\begin{aligned}
\left\{p: p_{i, j}>0, \sum_{j} p_{i, j}<1 \text { for any } 1 \leq i \leq n \text { and } 1 \leq j \leq m(i),\right. & \\
& j \neq i+1(\bmod n)\} .
\end{aligned}
$$

Now Lemma 2.4 can be used and the proof is finished.

It is an interesting problem to prove completeness of $F$ in a more general case. We shall treat it, using graph theory, in a next paper, under weaker assumptions about the set $\mathcal{Z}$.

\section{References}

[1] Denny, J. L., Yakowitz, S. J., Admissible run-contingency type tests for independence and Markov dependence, J. Amer. Statist. Assoc. 73 (1978), 177-181.

[2] Denny, J. L., Wright, A. L., On tests for Markov dependence, Z. Wahrsch. Verw. Gebiete 43(4) (1978), 331-338.

[3] Lehmann, E. L., Scheffe, H., Completeness, similar regions, and unbiased estimation. I, Sankhya 10 (1950), 305-340.

[4] Lehmann, E. L., Scheffe, H., Completeness, similar regions, and unbiased estimation. II, Sankhya 15 (1955), 219-236. 
[5] Lehmann, E. L., Testing Statistical Hypotheses, Wiley, New York, 1986.

[6] Paszkiewicz, A., When transition count for Markov chains is a complete sufficient statistic, Statist. Probab. Lett., to appear.

[7] Schervish, M. J., Theory of Statistics, Springer-Verlag, New York, 1995.

\section{AgnieszKa Palma}

FACULTy of Mathematics

UNIVERSITY OF ŁÓDŹ

BANACHA 22

90-238 ŁÓDŹ, POLAND

E-MAIL: APALMA@MATH.UNI.LODZ.PL 